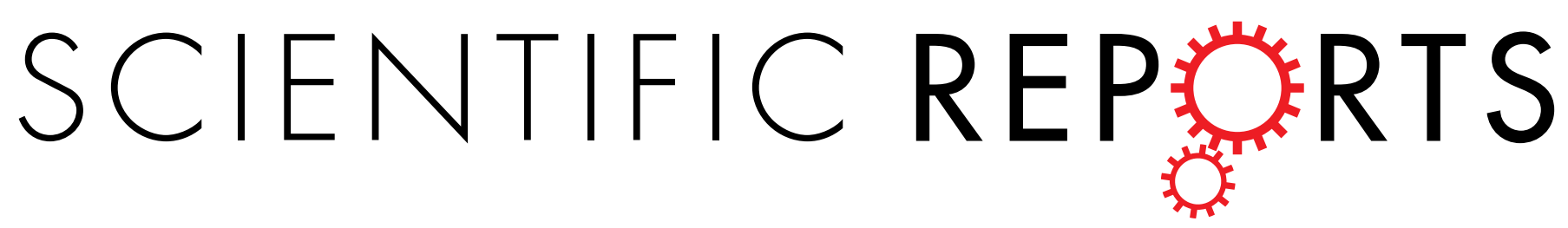

\title{
Erratum: Structural and Dynamic Features of F-recruitment Site Driven Substrate Phosphorylation
} by ERK2

Andrea Piserchio, Venkatesh Ramakrishan, Hsin Wang, Tamer S. Kaoud, Boris Arshava, Kaushik Dutta, Kevin N. Dalby \& Ranajeet Ghose

Scientific Reports 5:11127; doi: 10.1038/srep11127; published online 08 June 2015; updated on 31 July 2015 In the PDF version of this Article, Equation (2) contains typographical errors.

$$
\Delta_{i, s c}=100\left(\frac{\left|\delta_{0, H}-\delta_{i, H}\right|}{2 \sigma_{B M R B, H}}+\frac{\left|\delta_{0, h e t}-\delta_{i, h e t}\right|}{2 \sigma_{B M R B, h e t}}\right)
$$

should read:

$$
\Delta_{i, s c}=100\left(\frac{\left|\delta_{0, H}-\delta_{i, H}\right|}{\sigma_{B M R B, H}}+\frac{\left|\delta_{0, h e t}-\delta_{i, h e t}\right|}{\sigma_{B M R B, h e t}}\right)
$$

